\title{
Pause \& go: from the discovery of RNA polymerase pausing to its functional implications Andreas Mayer ${ }^{*}$, , , Heather M Landry ${ }^{*}$ and L Stirling Churchman
}

The synthesis of nascent RNA is a discontinuous process in which phases of productive elongation by RNA polymerase are interrupted by frequent pauses. Transcriptional pausing was first observed decades ago, but was long considered to be a special feature of transcription at certain genes. This view was challenged when studies using genome-wide approaches revealed that RNA polymerase II pauses at promoter-proximal regions in large sets of genes in Drosophila and mammalian cells. High-resolution genomic methods uncovered that pausing is not restricted to promoters, but occurs globally throughout gene-body regions, implying the existence of keyrate limiting steps in nascent RNA synthesis downstream of transcription initiation. Here, we outline the experimental breakthroughs that led to the discovery of pervasive transcriptional pausing, discuss its emerging roles and regulation, and highlight the importance of pausing in human development and disease.

\author{
Address \\ Department of Genetics, Harvard Medical School, Boston, MA, United \\ States \\ Corresponding author: Churchman, L Stirling (churchman@genetics. \\ med.harvard.edu) \\ *Equal contribution. \\ " Current address: Max Planck Institute for Molecular Genetics, Berlin, \\ Germany.
}

\author{
Current Opinion in Cell Biology 2017, 46:72-80 \\ This review comes from a themed issue on Cell nucleus \\ Edited by Aaron F. Straight and Roland Foisner \\ For a complete overview see the Issue and the Editorial \\ Available online 28th March 2017 \\ http://dx.doi.org/10.1016/j.ceb.2017.03.002 \\ 0955-0674/@ 2017 Elsevier Ltd. All rights reserved.
}

\section{Introduction}

During transcription, DNA-bound RNA polymerases synthesize nascent RNA molecules along a chromatin DNA template. This process is generally divided into three distinct phases: initiation, elongation and termination (Figure 1) [1,2]. For decades, transcription initiation was thought to be the major regulatory step in the production of RNA [3], but this view was recently challenged by the discovery of widespread RNA polymerase pausing during transcription elongation, suggesting that events downstream of transcription initiation, such as pausing in the promoter-proximal region [4,5], are also key rate-limiting steps $\left[6,7^{\circ}\right]$. Thus, nascent RNA synthesis is a discontinuous process, during which phases of productive transcription are frequently interrupted by pauses. Although the causes and consequences of transcriptional pausing remain incompletely understood, pausing of RNA polymerase clearly creates opportunities for regulation.

Over the past four decades, analysis of transcriptional pausing has been stimulated by multiple technological advances. To orient the reader, Box 1 provides an overview and comparison of methods used successfully to study transcriptional pausing in vivo. All of these experimental approaches reveal the position and relative abundance of RNA polymerase throughout gene bodies. In these data, peaks in RNA polymerase occupancy correspond to pause sites, because locations where RNA polymerase is captured more readily are positions where RNA polymerase spends more time on average (Figure 1). Additionally, these peaks of RNA polymerase are engaged transcription complexes as they are associated with nascent RNA and are capable of resuming transcription. In this review, we describe how technological advances have taken us from the discovery of RNA polymerase pausing to our emerging appreciation of its roles in regulating RNA abundance and identity.

Although various types of RNA polymerase pausing are mentioned, the focus of this review is on the emerging analysis of gene-body pausing by RNA polymerase II (Pol II), the key enzyme that produces all protein-coding RNAs and a large set of non-coding RNAs in eukaryotic cells [8-10]. We refer readers who are especially interested in Pol II promoter-proximal pausing to the following excellent reviews: [4,5,11].

\section{The road to the discovery of pervasive transcriptional pausing}

The first evidence of RNA polymerase pausing arose from in vitro transcription experiments performed in the early 1970s (Figure 2). In two seminal studies, Maizels et al. and Dahlberg et al. used in vitro transcription assays to show that Escherichia coli RNA polymerase transcribing the lac operon or lambda DNA transiently pauses at discrete sites [12-14]. This in vitro pausing was confirmed by multiple laboratories during the 1970 s and early 1980 s [15-17]. RNA polymerase pausing in vivo was first demonstrated in 1981 by the Chambon laboratory, who used a transcriptional run-on assay to show that Pol II accumulates at the $5^{\prime}$-end of the beta-globin gene in hen 


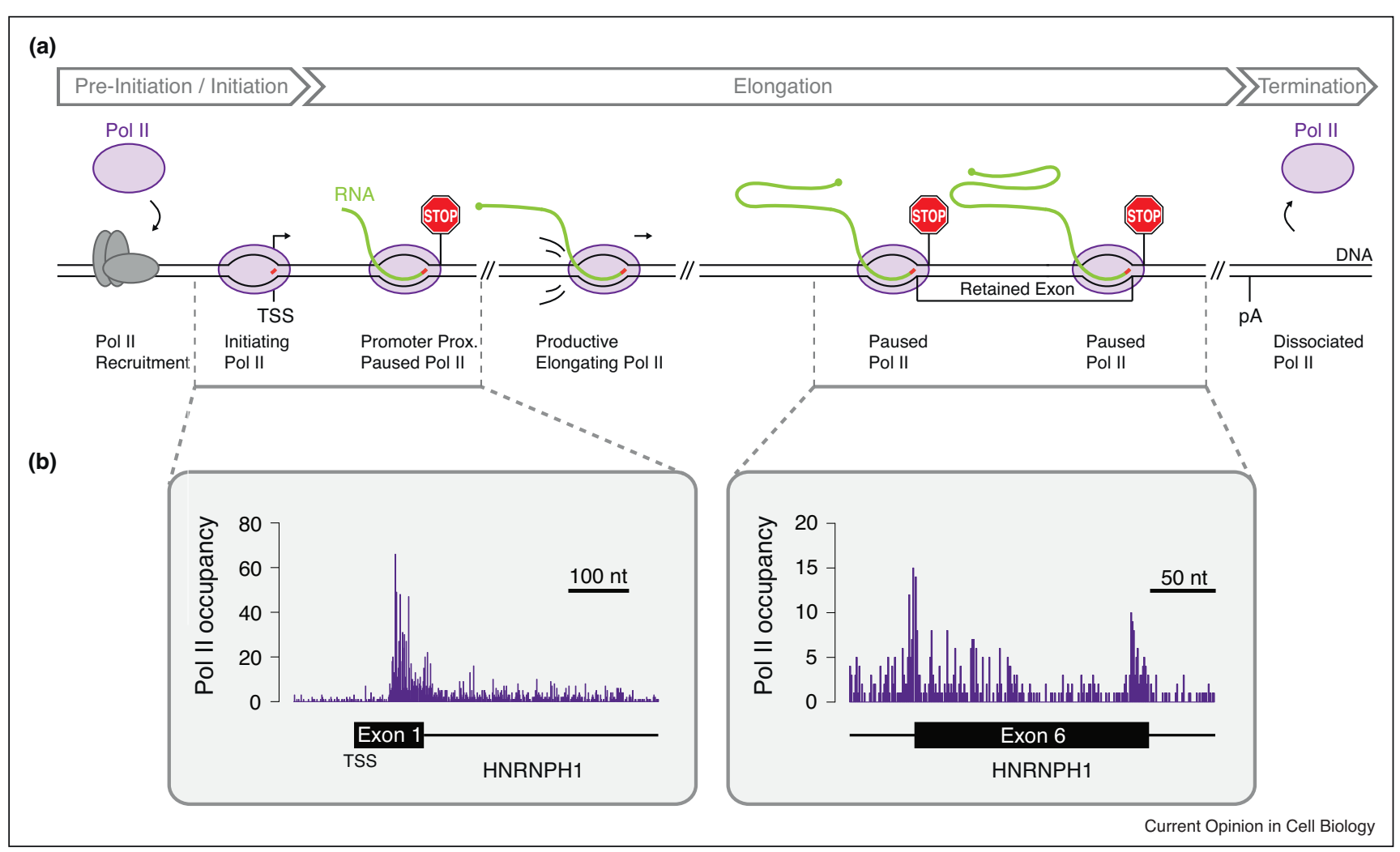

RNA polymerase II pausing during the gene transcription cycle.

(a) Different transcriptional states of RNA polymerase II (Pol II) during distinct phases of the gene transcription cycle: pre-initiation/initiation, elongation, and termination. Pol II is shown in purple; nascent RNA is in green with the $3^{\prime}$-end nucleotide labeled in red; double-stranded DNA is indicated by two black lines; TSS: transcription start site; PA: polyadenylation site.

(b) Pol II pausing with single-nucleotide resolution at the promoter-proximal (left panel) and gene-body regions (right panel) as revealed by human NET-seq (Box 1). NET-seq data obtained from HeLa S3 cells are shown for the representative HNRNPH1 gene [57 ${ }^{\circ}$ ]. Exonic and intronic regions are indicated by black boxes and black lines, respectively. TSS: transcription start site.

erythrocytes. This finding provided the first evidence for Pol II promoter-proximal pausing [18].

The advent of chromatin immunoprecipitation (ChIP) and improved transcription run-on protocols (see Box 1) in the mid-1980s enabled detection of Pol II accumulation at the $5^{\prime}$-ends of additional genes in Drosophila and mammalian cells. Studies performed in the Lis laboratory revealed that Pol II clusters within a small window at the $5^{\prime}$-end of the hsp70 heat shock gene in Drosophila [19], and that the accumulated polymerases are in a paused state [20]. Pol II accumulation at the $5^{\prime}$-ends of genes was also observed at selected mammalian genes, first in $c$-myc by the Groudine, Bornkamm and Marcu laboratories [2123], and subsequently in other genes such as $c$-myb, $c$-fos, $c$ mos and ADA [24]. Nuclear run-on experiments at the cmyc gene using short probes revealed that the majority of transcriptionally engaged Pol II was located within the first 169 nucleotides [25,26]. Together, these studies suggested that key rate-limiting steps occur downstream of initiation in a handful of genes.
The earliest insights into the molecular mechanisms underlying pausing were obtained from in vitro transcription experiments that revealed that certain DNA sequences cause RNA polymerase to move backward, a phenomenon known as backtracking [27]. Backtracking results in the misalignment of nascent RNA and the RNA polymerase active site, and can lead to a transcriptional arrest [27-31]. Release of RNA polymerase from a transcriptional arrest is aided by multiple trans-acting factors. The first transcription factor to be implicated in the regulation of transcriptional pausing was TFIIS [32,33]. TFIIS induces cleavage of the nascent RNA, restoring a $3^{\prime} \mathrm{OH}$ of the nascent RNA to the RNA polymerase active site, which is thought to enable the release of arrested Pol II [34-38].

Up to this point, the prevailing view in the field was that gene expression is primarily regulated at two main steps: recruitment of Pol II and transcription initiation. However, this model was challenged by the advent of genomewide Pol II mapping methods. The first genome-wide 
Box 1 Methods for analyzing RNA polymerase pausing in vivo.

\section{Chromatin immunoprecipitation (ChIP) of RNA polymerase:}

ChIP determines the position of RNA polymerase along genes and genomes in vivo [19,39,123]. Chromatin is reversibly crosslinked, typically with formaldehyde [124,125]. After chromatin fragmentation and solubilization, DNA bound by RNA polymerase is purified by immunoprecipitation. After reversal of the crosslinks, the relative amount of captured DNA is assessed by quantitative PCR (ChIP-qPCR), microarray hybridization (ChIP-chip) $[39,40]$, or high-throughput sequencing (ChIP-seq) [123]. Variations on ChIP-seq such as ChIP-exo $[50,126]$ and ChIP-nexus [61 $\left.{ }^{\circ}, 127^{\circ}\right]$, employ exonuclease digestion to remove DNA that is not directly bound by RNA polymerase, yielding nucleotide resolution and higher sensitivity.

ChIP-based approaches lack DNA strand specificity and suffer from high levels of background signals. Moreover, the spatial resolution of classical ChIP assays is limited, typically $>200 \mathrm{bp}$. Furthermore, because the bound DNA is analyzed rather than the nascent RNA, the transcriptional status of RNA polymerase is not revealed.

\section{Transcription run-on:}

Transcription run-on approaches exploit the nascent RNA to reveal the location of transcribing RNA polymerase [20,128]. In nuclear run-on assays, cells are lysed and nuclei are prepared, halting transcription by RNA polymerase. Transcription is then restarted in vitro for a short amount of time in the presence of labeled nucleotides. The amount of labeled, nascent RNA at a given region corresponds to the abundance of RNA polymerase, providing a measure of RNA polymerase occupancy at selected genes. Two genome-wide transcription run-on methods have been developed to assess RNA polymerase density: global run-on sequencing (GRO-seq) [51] and precision nuclear run-on and sequencing (PRO-seq) [55,56,129*]. In both techniques, labeled nascent RNA is purified, converted into a DNA sequencing library, and sequenced. PRO-seq is a variant of GRO-seq that reveals the RNA polymerase density at single-nucleotide resolution $[55,56]$.

Transcription-run on assays provide a strand-specific quantitative measure of transcriptionally engaged RNA polymerase. However, these methods rely on the efficient restart of transcription under non-physiological conditions, which depends on the experimental procedure and the transcriptional status of RNA polymerase [130]. Notably, arrested, backtracked RNA polymerase cannot be restarted, and will therefore escape detection [79].

\section{Permanganate footprinting:}

This approach detects single-stranded DNA regions as they occur in the transcription bubble of transcriptionally engaged RNA polymerase [131] by exploiting the hyper-reactivity of thymine residues within single-stranded DNA to oxidation by potassium permanganate. After permanganate treatment, the DNA is specifically cleaved at oxidized thymines, and the positions of resulting DNA breaks are determined by ligation-mediated PCR (LM-PCR). This approach was recently adapted for genome-wide analysis [132].

Permanganate footprinting is intended to detect single-stranded DNA within the RNA polymerase transcription bubble. However, single-stranded DNA which arise from other sources, such as DNA replication forks and R-loops, contributes to the experimental background.

\section{Native elongating transcript sequencing (NET-seq):}

In NET-seq, the $3^{\prime}$-ends of nascent RNAs are detected by to high-throughput sequencing, thereby revealing both the genomic location and abundance of RNA polymerase $\left[133,134^{\circ}, 135^{\circ}\right]$. Nascent RNA is co-purified with the extremely stable RNA-DNA-RNA polymerase ternary complex, either by immunoprecipitation or cell fractionation. Because transcriptional restart in vitro is not required, all RNA polymerases can be monitored, regardless of their pausing status. The $3^{\prime}$-ends of nascent RNAs are converted into a DNA sequencing library, and the identity and abundance of the library fragments are determined by deep sequencing. Mapping the sequencing reads to the reference genome reveals the strand-specific genomic position of the RNA polymerase active site with single-nucleotide precision.

In addition to the $3^{\prime}$-ends of nascent RNAs, NET-seq libraries capture RNA processing intermediates from splicing and $3^{\prime}$-end cleavage, as well as fully processed mature RNAs. These background sequences can be computationally identified and removed, but processing the data in this manner decreases the fraction of informative reads.

approach that has been used to study transcriptional pausing was ChIP coupled with microarray hybridization (ChIP-chip), initially developed in the mid-2000s [39,40] (Box 1). This experimental tool revealed that Pol II clusters in the promoter-proximal regions of a large set of active genes in various model organisms [41-44]. ChIPchip, and subsequently ChIP coupled with deep sequencing (ChIP-seq), revealed that Pol II accumulation is not restricted to $5^{\prime}$-ends of genes, but also occurs globally at $3^{\prime}$-ends $\left[45-48,49^{\bullet \bullet}\right]$. With a high-resolution ChIP study, ChIP-exo (Box 1), Pol II was observed to accumulate on average $50 \mathrm{bp}$ downstream of the TSS at the majority of genes in human K562 cells [50].

Although these genomic Pol II ChIP studies suggested that peaks in Pol II density are widespread, the transcriptional status of Pol II, that is whether it is engaged in transcription, was uncertain. Critically, it remained unclear whether the clusters of Pol II corresponded to paused Pol II. This limitation was initially overcome by combining genomic ChIP with potassium permanganate footprinting assays at selected genes (Box 1) $[41,42]$ and later by the development of methods for mapping the locations of transcriptionally engaged Pol II throughout the genome, for example, global run-on sequencing [51] (GRO-seq, Box 1), as well as sequencing of short and capped RNAs (scRNA-seq) [52,53 ${ }^{\circ}$.

Recently, techniques for mapping Pol II at nucleotide resolution throughout the genome have enabled pausing to be analyzed across gene bodies, outside of promoterproximal regions. Native elongating transcript sequencing 


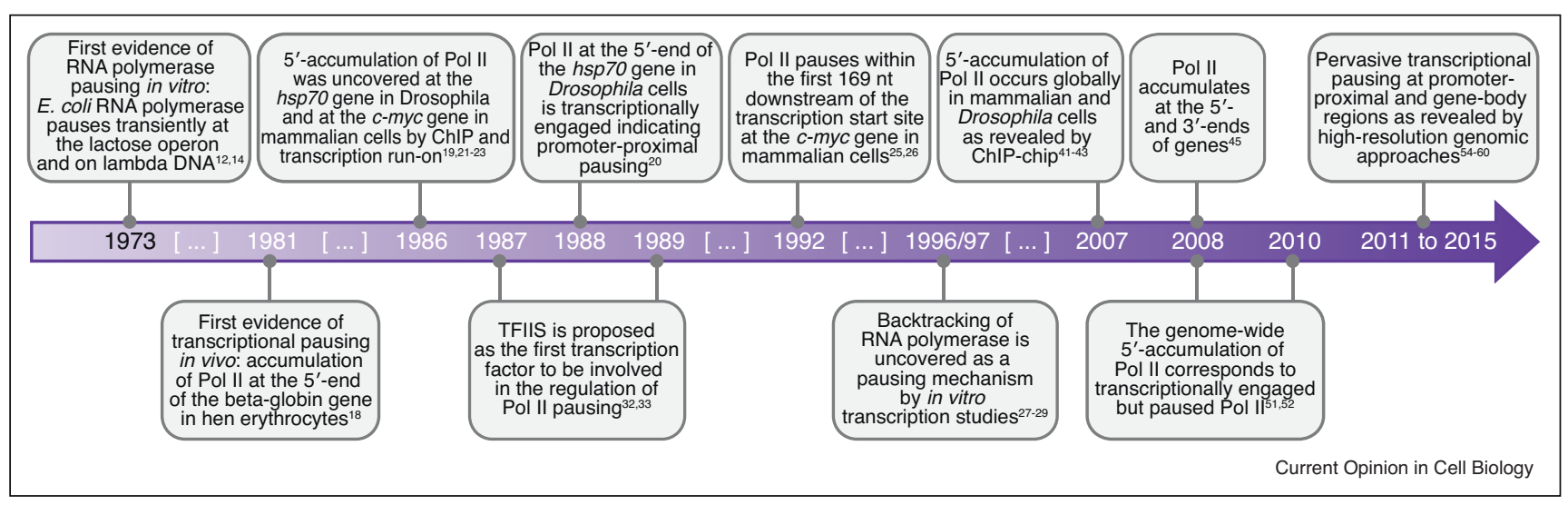

Milestones on the road to the discovery of pervasive transcriptional pausing.

(NET-seq, Box 1) and precision nuclear run-on and sequencing (PRO-seq, Box 1) revealed that RNA polymerase pausing occurs throughout gene-body regions in various model organisms, ranging from bacteria to human cells [54$\left.56,57^{\bullet \bullet}, 58^{\bullet \bullet}, 59,60\right]$. These high-resolution techniques reveal that in yeast and bacteria, RNA polymerase pauses approximately every $20-100$ basepairs $[54,59]$. These studies determined that the relative magnitude of promoterproximal pausing varies between genes, and in many cases Pol II density in the region around the TSS is more than 10fold higher than the rest of the gene body $\left[55,57^{\bullet \bullet}\right]$. Nevertheless, genome-wide Pol II density averages show that prominent pauses occur at the boundaries of retained exons and near polyadenylation sites $\left[55,57^{\bullet \bullet}, 58^{\bullet \bullet}, 61^{\bullet \bullet}\right]$.

Together, these studies provide strong evidence for pervasive RNA polymerase pausing and imply that it represents a general feature of gene transcription (Figure 2). Efforts currently underway in our group and others aim to better understand the causes and functional roles of transcriptional pausing.

\section{Causes of RNA polymerase pausing}

Diverse mechanisms modulate the frequency and lifetime of RNA polymerase pauses. Many in vitro biochemical and biophysical studies have revealed the detailed molecular mechanisms that are responsible for Pol II pausing and are reviewed elsewhere $[62,63]$. Whether in vivo transcriptional pausing occurs through these same biophysical mechanisms is not clear, but it is likely that many pauses exploit them.

RNA polymerase has an intrinsic capacity for pausing and alterations in any component of the RNA-DNA-RNA polymerase ternary complex can trigger this natural propensity to pause. Specific amino acid residues in Pol II, particularly in the trigger loop domain, are critical for transcription elongation and pausing. Mutating these residues leads to an increase or decrease in the transcription elongation rate and tendency to pause [64-66]. Certain DNA sequences, on their own, cause RNA polymerase pausing $[12,16,67]$. For example, regions around promoters and termination sites often have high $\mathrm{G} / \mathrm{C}$ content followed by A/T-rich sequences $\left[52,68^{\circ}\right]$. This sequence feature is thought to induce RNA polymerase backtracking and pausing by abruptly reducing the melting temperature and stability of the DNA-RNA hybrid that forms within the transcription elongation complex $[69,70]$. Other sequence elements associated with transcriptional pausing include 'A' nucleotides, which are present at sites of pausing in Saccharomyces cerevisiae [54], and a 12-bp consensus sequence detected at E. coli pause sites both in vivo $\left[59,60,71^{\circ}\right]$ and in vitro $[59,67]$. Nascent RNA can also induce pausing by forming either secondary structures or R-loops that interfere with the transcription elongation complex. For example, RNA hairpins that form within or upstream of the RNA exit channel cause pausing $\left[30,72^{\circ}\right]$. R-loops are also implicated in Pol II pausing during transcription termination [73,74]. Thus, each of the core components of transcription - Pol II, DNA, and RNA - are intrinsically capable of inducing pausing.

Extrinsic factors, such as nucleosomes and regulatory factors, are also capable of inducing RNA polymerase pausing. For most of these, the biophysical mechanisms by which Pol II pausing occurs are not known, however we propose that extrinsic factors likely act by perturbing some aspect of the RNA-DNA-RNA polymerase ternary complex. In other words, the biophysical mechanisms for pausing outlined above are likely utilized when extrinsic factors manipulate Pol II elongation.

Nucleosomes, which compact and organize chromatin, present a significant barrier to transcription. In vitro transcription through nucleosomes is punctuated by 
increased pausing compared to naked DNA [75], which can be alleviated by elongation factors such as TFIIS [76,77] and histone chaperones such as FACT [78]. Nucleosomes also hamper transcription in vivo $[54,55,79]$, but to a lesser degree due to the presence of chromatin remodelers $[80,81]$ and transcription elongation factors [79]. Consistent with the idea that nucleosomes represent a transcriptional barrier, depletion of histones from human cells increases the speed of transcription elongation $\left[82^{\bullet \bullet}\right]$.

Beyond the chromatin itself, dozens of regulatory factors interact with the transcription elongation complex as it moves along a gene $\left[46,61^{\bullet \bullet}, 83-87\right]$, and many influence pausing and transcription elongation rates. Promoterproximal pausing is regulated in part by negative elongation factors such as NELF and DSIF, which interact with Pol II to induce pausing by a mechanism that has yet to be revealed (for review, see Ref. [88]). DNA binding factors, such as CTCF, YY1, and Reb1, are thought to induce Pol II pausing by creating 'roadblocks' on the DNA template that obstruct transcription elongation $\left[57^{\bullet \bullet}, 89,90^{\circ \bullet}\right]$. Many features of these pausing factors remain to be elucidated, but their existence demonstrates that transcriptional pausing is highly regulated and likely a core component of diverse gene regulatory mechanisms.

\section{Emerging roles of transcriptional pausing}

RNA polymerase pausing occurs throughout transcription elongation. Even though the precise biophysical mechanisms of in vivo Pol II pausing have yet to be established for each step in the transcription cycle, a number of functions for pausing have been proposed [63]. The main consequence of Pol II pausing are the windows of opportunity for regulation and coordination with other processes at different stages during transcription elongation.

During early transcription elongation, promoter-proximal pausing represents a key rate-limiting step in RNA synthesis that can impact mRNA abundance [91-93]. Promoter-proximal pausing is also thought to control gene expression by maintaining an open chromatin state near promoters [94-96]. Additionally, RNA polymerase pausing is implicated in diverse gene regulatory mechanisms, including coupling between transcription and translation in bacteria $[59,97]$, suppressing genome instability $\left[98^{\bullet \bullet}\right]$, and promoting RNA folding [99].

Transcriptional pausing also occurs downstream of the promoter-proximal region. A great deal of work over the past decade has suggested that pausing plays a role in splicing. Splicing often occurs co-transcriptionally, while the nascent RNA is attached to chromatin [100], and in close proximity to transcribing Pol II $\left[101^{\circ}\right]$. The proximity between the splicing and transcription machineries suggests that transcription elongation could impact splicing through kinetic coupling since the pausing of Pol II creates a 'window of opportunity' for splicing to occur (proposed by Refs. [102-104] and recently reviewed in Refs. $[105,106])$. Several lines of evidence support this kinetic coupling model. First, mutations in Pol II that alter the transcription elongation rate cause widespread changes in alternative splicing [107-110], suggesting that proper transcription rate is important for alternative splicing regulation. Second, Pol II density is elevated around splice sites $\left[55,57^{\bullet \bullet}, 58^{\bullet \bullet}, 61^{\bullet \bullet}, 111\right]$. Third, Pol II pausing induced by the transcription factor, CTCF, can cause retention of weak exons, as demonstrated in the $C D 45$ gene [89].

At the $3^{\prime}$-ends of protein-coding genes, Pol II must be released from DNA in order to be recycled and to prevent read-through transcription into downstream genes. Pol II density increases after polyadenylation sites [45$\left.48,49^{\bullet \bullet}, 51\right]$ and at putative termination sites [68 ${ }^{\bullet \bullet}$ ], suggesting that transcription termination requires pausing or slowing [112]. Consistent with this model, Pol II mutants with altered transcription elongation rates display different sites of transcription termination $\left[113,114^{\circ}\right]$. The proposed causes of RNA polymerase pausing during termination in eukaryotes include Pol II backtracking $\left[68^{\bullet \bullet}, 115,116^{\bullet \bullet}\right]$, R-loop formation [73,74], and Reb1mediated transcription blockage $\left[90^{\circ} \bullet\right.$.

Analysis of the functional roles of transcriptional pausing is still in its infancy. Future work will likely uncover a broad spectrum of mechanisms that manipulate Pol II elongation, particularly in relation to disease development. Promoter-proximal pausing and aberrant pause release have been implicated in various human pathologies $\left[117^{\circ}\right]$ such as in c-Myc driven cancers $[25,118]$ and cardiac hypertrophy [119], and also plays a role in inflammatory responses [120], as well as in development $[11,121]$. Therefore, we expect other classes of transcriptional pausing, for example the elevated polymerase density around splice sites and polyadenylation signals, to also play critical roles in human pathologies.

\section{Future directions}

Following the advent of high-resolution tools for analyzing RNA polymerase pausing throughout the genome, the next steps are to elucidate the regulation and function of these pauses in normal conditions and in disease. To achieve this, additional tools will be necessary. First, we need better methods for inducing transcriptional pausing. RNA polymerase mutants with altered transcription elongation rates have contributed greatly to our understanding of the connections between transcription elongation rates and other processes. However, little mechanistic insight has been gleaned from these systems because the impact of these mutations on transcriptional pausing remains poorly characterized. Altering DNA sequence to induce transcriptional pausing or to impact the binding of a roadblock protein is a necessary complementary approach 
to uncovering the functional roles of RNA polymerase pausing. Second, we need to resolve the temporal order of RNA polymerase pausing in relation to the arrival of regulatory factors and RNA processing machinery, in order to determine whether pausing kinetics truly influence the window of opportunity for co-transcriptional events. Finally, imaging studies have shown that in vivo elongation rates vary significantly among individual Pol II enzymes [122]. Thus, it will be important to understand whether transcriptional pausing is a purely stochastic event or differentially regulated for individual Pol II enzymes.

\section{Acknowledgements}

We thank F. Winston, E. Altendorfer, C. Patil and A. Snavely for critical comments on the manuscript. This work was supported by US National Institutes of Health National Human Genome Research Institute (NHGRI) grant R01HG007173 and National Institutes of General Medical Science (NIGMS) grant R01GM117333 to L.S.C.; and a Burroughs Wellcome Fund Career Award at the Scientific Interface (to L.S.C.). A.M. was supported by the Long-Term Postdoctoral Fellowships of the Human Frontier Science Program (HFSP) (LT000314/2013-L) and the European Molecular Biology Organization (EMBO) (ALTF858-2012).

\section{References and recommended reading}

Papers of particular interest, published within the period of review, have been highlighted as:

- of special interest

$\bullet$ of outstanding interest

1. Shandilya J, Roberts SGE: The transcription cycle in eukaryotes: from productive initiation to RNA polymerase II recycling. Biochim Biophys Acta 2012, 1819:391-400.

2. Svejstrup JQ: The RNA polymerase II transcription cycle: cycling through chromatin. Biochim Biophys Acta 2004, 1677:64-73.

3. Ptashne M, Gann A: Transcriptional activation by recruitment. Nature 1997, 386:569-577.

4. Adelman K, Lis JT: Promoter-proximal pausing of RNA polymerase II: emerging roles in metazoans. Nat Rev Genet 2012, 13:720-731.

5. Li J, Gilmour DS: Promoter proximal pausing and the control of gene expression. Curr Opin Genet Dev 2011, 21:231-235.

6. Margaritis T, Holstege FCP: Poised RNA polymerase II gives pause for thought. Cell 2008, 133:581-584.

7. Jonkers I, Lis JT: Getting up to speed with transcription

- elongation by RNA polymerase II. Nat Rev Mol Cell Biol 2015, 16:167-177.

This excellent review on transcription elongation focuses on the regulation of promoter-proximal pausing by Pol II.

8. Djebali S, Davis CA, Merkel A, Dobin A, Lassmann T, Mortazavi A et al.: Landscape of transcription in human cells. Nature 2012 489:101-108.

9. Jensen $\mathrm{TH}$, Jacquier A, Libri D: Dealing with pervasive transcription. Mol Cell 2013, 52:473-484.

10. Pelechano V, Steinmetz LM: Gene regulation by antisense transcription. Nat Rev Genet 2013, 14:880-893.

11. Gaertner B, Zeitlinger J: RNA polymerase II pausing during development. Development 2014, 141:1179-1183.

12. Maizels NM: The nucleotide sequence of the lactose messenger ribonucleic acid transcribed from the UV5 promoter mutant of Escherichia coli. Proc Natl Acad Sci U S A 1973, 70:3585-3589.
13. Gilbert W, Maizels N, Maxam A: Sequences of controlling regions of the lactose operon. Cold Spring Harb Symp Quant Biol 1974, 38:845-855.

14. Dahlberg JE, Blattner FR: In vitro transcription products of lambda DNA: nucleotide sequences and regulatory sites. In Virus Research. Edited by Fox CF, Robinson WS. New York: Academic Press; 1973:533-544.

15. Lee F, Squires CL, Squires C, Yanofsky C: Termination of transcription in vitro in the Escherichia coli tryptophan operon leader region. J Mol Biol 1976, 103:383-393.

16. Kassavetis GA, Chamberlin MJ: Pausing and termination of transcription within the early region of bacteriophage T7 DNA in vitro. J Biol Chem 1981, 256:2777-2786.

17. Kingston RE, Chamberlin MJ: Pausing and attenuation of in vitro transcription in the rrnB operon of E. coli. Cell 1981, 27:523-531.

18. Gariglio $\mathrm{P}$, Bellard M, Chambon P: Clustering of RNA polymerase $B$ molecules in the $5^{\prime}$ moiety of the adult beta-globin gene of hen erythrocytes. Nucleic Acids Res 1981, 9:2589-2598.

19. Gilmour DS, Lis JT: RNA polymerase II interacts with the promoter region of the noninduced hsp70 gene in Drosophila melanogaster cells. Mol Cell Biol 1986, 6:3984-3989.

20. Rougvie AE, Lis JT: The RNA polymerase II molecule at the $5^{\prime}$ end of the uninduced hsp70 gene of D. melanogaster is transcriptionally engaged. Cell 1988, 54:795-804.

21. Bentley DL, Groudine M: A block to elongation is largely responsible for decreased transcription of c-myc in differentiated HL60 cells. Nature 1986, 321:702-706.

22. Eick D, Bornkamm GW: Transcriptional arrest within the first exon is a fast control mechanism in c-myc gene expression. Nucleic Acids Res 1986, 14:8331-8346.

23. Nepveu A, Marcu KB: Intragenic pausing and anti-sense transcription within the murine c-myc locus. EMBO J 1986, 5:2859-2865.

24. Spencer CA, Groudine M: Transcription elongation and eukaryotic gene regulation. Oncogene 1990, 5:777-785.

25. Strobl LJ, Eick D: Hold back of RNA polymerase II at the transcription start site mediates down-regulation of c-myc in vivo. EMBO J 1992, 11:3307-3314.

26. Krumm A, Meulia T, Brunvand M, Groudine M: The block to transcriptional elongation within the human c-myc gene is determined in the promoter-proximal region. Genes Dev 1992, 6:2201-2213.

27. Nudler E, Mustaev A, Lukhtanov E, Goldfarb A: The RNA-DNA hybrid maintains the register of transcription by preventing backtracking of RNA polymerase. Cell 1997, 89:33-41.

28. Reeder TC, Hawley DK: Promoter proximal sequences modulate RNA polymerase II elongation by a novel mechanism. Cell 1996, 87:767-777.

29. Komissarova N, Kashlev M: RNA polymerase switches between inactivated and activated states by translocating back and forth along the DNA and the RNA. J Biol Chem 1997, 272:1532915338.

30. Artsimovitch I, Landick R: Pausing by bacterial RNA polymerase is mediated by mechanistically distinct classes of signals. Proc Natl Acad Sci U S A 2000, 97:7090-7095.

31. Cheung ACM, Cramer P: Structural basis of RNA polymerase II backtracking, arrest and reactivation. Nature 2011, 471:249-253.

32. Reinberg D, Roeder RG: Factors involved in specific transcription by mammalian RNA polymerase II. Transcription factor IIS stimulates elongation of RNA chains. J Biol Chem 1987, 262:3331-3337.

33. Reines D, Chamberlin MJ, Kane CM: Transcription elongation factor SII (TFIIS) enables RNA polymerase II to elongate 
through a block to transcription in a human gene in vitro. $J$ Biol Chem 1989, 264:10799-10809.

34. Reines D: Elongation factor-dependent transcript shortening by template-engaged RNA polymerase II. J Biol Chem 1992, 267:3795-3800.

35. Izban MG, Luse DS: The RNA polymerase II ternary complex cleaves the nascent transcript in a $3^{\prime} \rightarrow 5^{\prime}$ direction in the presence of elongation factor SII. Genes Dev 1992, 6:1342-1356.

36. Wang D, Hawley DK: Identification of a $3^{\prime} \rightarrow 5^{\prime}$ exonuclease activity associated with human RNA polymerase II. Proc Natl Acad Sci U S A 1993, 90:843-847.

37. Gu W, Reines D: Variation in the size of nascent RNA cleavage products as a function of transcript length and elongation competence. J Biol Chem 1995, 270:30441-30447.

38. Kettenberger H, Armache K-J, Cramer P: Architecture of the RNA polymerase II-TFIIS complex and implications for mRNA cleavage. Cell 2003, 114:347-357.

39. Buck MJ, Lieb JD: ChIP-chip: considerations for the design, analysis, and application of genome-wide chromatin immunoprecipitation experiments. Genomics 2004, 83:349-360.

40. Kim TH, Barrera LO, Zheng M, Qu C, Singer MA, Richmond TA et al.: A high-resolution map of active promoters in the human genome. Nature 2005, 436:876-880.

41. Muse GW, Gilchrist DA, Nechaev S, Shah R, Parker JS, Grissom SF et al:: RNA polymerase is poised for activation across the genome. Nat Genet 2007, 39:1507-1511.

42. Zeitlinger J, Stark A, Kellis M, Hong J-W, Nechaev S, Adelman K et al.: RNA polymerase stalling at developmental control genes in the Drosophila melanogaster embryo. Nat Genet 2007, 39:1512-1516.

43. Guenther MG, Levine SS, Boyer LA, Jaenisch R, Young RA: A chromatin landmark and transcription initiation at most promoters in human cells. Cell 2007, 130:77-88.

44. Radonjic M, Andrau J-C, Lijnzaad P, Kemmeren P, Kockelkorn TTJP, van Leenen D et al.: Genome-wide analyses reveal RNA polymerase II located upstream of genes poised for rapid response upon S. cerevisiae stationary phase exit. Mol Cell 2005, 18:171-183.

45. Glover-Cutter K, Kim S, Espinosa J, Bentley DL: RNA polymerase II pauses and associates with pre-mRNA processing factors at both ends of genes. Nat Struct Mol Biol 2008, 15:71-78.

46. Mayer A, Lidschreiber M, Siebert M, Leike K, Söding J, Cramer P: Uniform transitions of the general RNA polymerase II transcription complex. Nat Struct Mol Biol 2010, 17:1272-1278.

47. Bataille AR, Jeronimo C, Jacques P-É, Laramée L, Fortin M-Ė, Forest A et al.: A universal RNA polymerase II CTD cycle is orchestrated by complex interplays between kinase, phosphatase, and isomerase enzymes along genes. Mol Cell 2012, 45:158-170.

48. Ferrari F, Plachetka A, Alekseyenko AA, Jung YL, Ozsolak F, Kharchenko PV et al.: "Jump start and gain" model for dosage compensation in Drosophila based on direct sequencing of nascent transcripts. Cell Rep 2013, 5:629-636.

49. Laitem C, Zaborowska J, Isa NF, Kufs J, Dienstbier M, Murphy S:

-. CDK9 inhibitors define elongation checkpoints at both ends of RNA polymerase II-transcribed genes. Nat Struct Mol Biol 2015, 22:396-403.

Global P-TEFb inhibition provides evidence for regulatory checkpoints at early and late stages during Pol II transcription elongation in human cells.

50. Pugh BF, Venters BJ: Genomic organization of human transcription initiation complexes. PLoS One 2016, 11: e0149339.

51. Core LJ, Waterfall JJ, Lis JT: Nascent RNA sequencing reveals widespread pausing and divergent initiation at human promoters. Science 2008, 322:1845-1848.
52. Nechaev S, Fargo DC, dos Santos G, Liu L, Gao Y, Adelman K: Global analysis of short RNAs reveals widespread promoterproximal stalling and arrest of Pol II in Drosophila. Science 2010, 327:335-338.

53. Scruggs BS, Gilchrist DA, Nechaev S, Muse GW, Burkholder A,

- Fargo DC et al.: Bidirectional transcription arises from two distinct hubs of transcription factor binding and active chromatin. Mol Cell 2015, 58:1101-1112.

Sequencing of short, $5^{\prime}$ capped nascent RNAs by START-seq reveals that pausing of Pol II in the sense and antisense directions around promoters defines the nucleosome-depleted region in mice.

54. Churchman LS, Weissman JS: Nascent transcript sequencing visualizes transcription at nucleotide resolution. Nature 2011 , 469:368-373.

55. Kwak H, Fuda NJ, Core LJ, Lis JT: Precise maps of RNA polymerase reveal how promoters direct initiation and pausing. Science 2013, 339:950-953.

56. Wang IX, Core LJ, Kwak H, Brady L, Bruzel A, McDaniel L et al.: RNA-DNA differences are generated in human cells within seconds after RNA exits polymerase II. Cell Rep 2014, 6:906-915.

57. Mayer A, di lulio J, Maleri S, Eser U, Vierstra J, Reynolds A et al.:

-• Native elongating transcript sequencing reveals human transcriptional activity at nucleotide resolution. Cell 2015, 161:541-554.

Adapting NET-seq to human cells reveals nucleotide-resolution Pol II pausing at splice junctions of retained exons and convergent transcription near gene promoters.

58. Nojima T, Gomes T, Grosso ARF, Kimura H, Dye MJ, Dhir S et al.: -• Mammalian NET-Seq reveals genome-wide nascent transcription coupled to RNA processing. Cell 2015, 161:526540.

Mammalian NET-seq using CTD phosphorylation-specific Pol II antibodies reveals discrete nascent RNA profiles for each Pol II CTD isoform.

59. Larson MH, Mooney RA, Peters JM, Windgassen T, Nayak D, Gross CA et al.: A pause sequence enriched at translation start sites drives transcription dynamics in vivo. Science 2014, 344:1042-1047.

60. Vvedenskaya IO, Vahedian-Movahed H, Bird JG, Knoblauch JG, Goldman SR, Zhang Y et al: Interactions between RNA polymerase and the "core recognition element" counteract pausing. Science 2014, 344:1285-1289.

61. Harlen KM, Trotta KL, Smith EE, Mosaheb MM, Fuchs SM,

-• Churchman LS: Comprehensive RNA polymerase II interactomes reveal distinct and varied roles for each phospho-CTD residue. Cell Rep 2016, 15:2147-2158.

Mass spectrometry analysis of Pol II CTD interactome in yeast uncovers new relationships between Pol II CTD interactions and co-transcriptional processes.

62. Imashimizu M, Shimamoto N, Oshima T, Kashlev M: Transcription elongation. Heterogeneous tracking of RNA polymerase and its biological implications. Transcription 2014, 5:e28285.

63. Landick R: The regulatory roles and mechanism of transcriptional pausing. Biochem Soc Trans 2006, 34:1062-1066.

64. Malagon F, Kireeva ML, Shafer BK, Lubkowska L, Kashlev M, Strathern JN: Mutations in the Saccharomyces cerevisiae RPB1 gene conferring hypersensitivity to 6-azauracil. Genetics 2006, 172:2201-2209.

65. Kaplan CD, Larsson K-M, Kornberg RD: The RNA polymerase II trigger loop functions in substrate selection and is directly targeted by alpha-amanitin. Mol Cell 2008, 30:547-556.

66. Kaplan CD, Jin H, Zhang IL, Belyanin A: Dissection of pol II trigger loop function and pol II activity-dependent control of start site selection in vivo. PLoS Genet 2012, 8:e1002627.

67. Herbert KM, La Porta A, Wong BJ, Mooney RA, Neuman KC, Landick $\mathrm{R}$ et al.: Sequence-resolved detection of pausing by single RNA polymerase molecules. Cell 2006, 125:1083-1094. 
68. Schwalb B, Michel M, Zacher B, Frühauf K, Demel C, Tresch A

-• et al:: TT-seq maps the human transient transcriptome. Science 2016, 352:1225-1228.

Sequencing of fragmented RNA after a short pulse of metabolic labeling provides new insights into the DNA sequence elements that control transcription termination.

69. Kireeva ML, Komissarova N, Waugh DS, Kashlev M: The 8nucleotide-long RNA:DNA hybrid is a primary stability determinant of the RNA polymerase II elongation complex. J Biol Chem 2000, 275:6530-6536.

70. Kulish D, Struhl K: TFIIS enhances transcriptional elongation through an artificial arrest site in vivo. Mol Cell Biol 2001, 21:4162-4168

71. Imashimizu M, Takahashi H, OshimaT Mclntosh C, Bubunenko M

- Court DL et al.: Visualizing translocation dynamics and nascent transcript errors in paused RNA polymerases in vivo. Genome Biol 2015, 16:98.

Combining NET-seq and RNase footprinting in E. coli, this study uncovers a consensus sequence associated with RNA polymerase pausing and also provides evidence for the importance of Gre factors in proofreading errors during transcription elongation.

72. Zhang J, Landick R: A two-way street: regulatory interplay

- between RNA polymerase and nascent RNA structure. Trends Biochem Sci 2016, 41:293-310.

This review outlines the relationship between nascent RNA structure and transcription by RNA polymerase.

73. Mischo HE, Gómez-González B, Grzechnik P, Rondón AG, Wei W, Steinmetz $L$ et al.: Yeast Sen1 helicase protects the genome from transcription-associated instability. Mol Cell 2011, 41:2132.

74. Skourti-Stathaki K, Proudfoot NJ, Gromak N: Human senataxin resolves RNA/DNA hybrids formed at transcriptional pause sites to promote Xrn2-dependent termination. Mol Cell 2011, 42:794-805.

75. Chang $\mathrm{CH}$, Luse DS: The $\mathrm{H} 3 / \mathrm{H} 4$ tetramer blocks transcript elongation by RNA polymerase II in vitro. J Biol Chem 1997 , 272:23427-23434

76. Kireeva ML, Hancock B, Cremona GH, Walter W, Studitsky VM, Kashlev M: Nature of the nucleosomal barrier to RNA polymerase II. Mol Cell 2005, 18:97-108.

77. Luse DS, Spangler LC, Újvári A: Efficient and rapid nucleosome traversal by RNA polymerase II depends on a combination of transcript elongation factors. J Biol Chem 2011, 286:6040-6048.

78. Orphanides G, LeRoy G, Chang CH, Luse DS, Reinberg D: FACT, a factor that facilitates transcript elongation through nucleosomes. Cell 1998, 92:105-116.

79. Weber CM, Ramachandran S, Henikoff S: Nucleosomes are context-specific, H2A.Z-modulated barriers to RNA polymerase. Mol Cell 2014, 53:819-830.

80. Skene PJ, Hernandez AE, Groudine M, Henikoff S: The nucleosomal barrier to promoter escape by RNA polymerase II is overcome by the chromatin remodeler Chd1. Elife 2014, 3 : e02042.

81. Venkatesh S, Workman JL: Histone exchange, chromatin structure and the regulation of transcription. Nat Rev Mol Cell Biol 2015, 16:178-189.

82. Jimeno-González S, Payán-Bravo L, Muñoz-Cabello AM, Guijo M,

-• Gutierrez G, Prado F et al.: Defective histone supply causes changes in RNA polymerase II elongation rate and cotranscriptional pre-mRNA splicing. Proc Natl Acad Sci U S A 2015, 112:14840-14845

Knockdown of stem-loop binding protein (SLBP), which reduces the levels of available histones, increases transcription elongation speed and alters splice isoforms.

83. Pokholok DK, Hannett NM, Young RA: Exchange of RNA polymerase II initiation and elongation factors during gene expression in vivo. Mol Cell 2002, 9:799-809.

84. Tardiff DF, Abruzzi KC, Rosbash M: Protein characterization of Saccharomyces cerevisiae RNA polymerase II after in vivo cross-linking. Proc Natl Acad Sci U S A 2007, 104:19948-19953.
85. Mosley AL, Sardiu ME, Pattenden SG, Workman JL, Florens L, Washburn MP: Highly reproducible label free quantitative proteomic analysis of RNA polymerase complexes. Mol Cell Proteomics 2011, 10 M110.000687.

86. Mosley AL, Hunter GO, Sardiu ME, Smolle M, Workman JL, Florens L et al.: Quantitative proteomics demonstrates that the RNA polymerase II subunits Rpb4 and Rpb7 dissociate during transcriptional elongation. Mol Cell Proteomics 2013, 12:1530-1538.

87. Harlen KM, Churchman LS: Subgenic Pol II interactomes identify region-specific transcription elongation regulators. Mol Syst Biol 2017, 13:900.

88. Kwak H, Lis JT: Control of transcriptional elongation. Annu Rev Genet 2013, 47:483-508

89. Shukla S, Kavak E, Gregory M, Imashimizu M, Shutinoski B, Kashlev $\mathrm{M}$ et al.: CTCF-promoted RNA polymerase II pausing links DNA methylation to splicing. Nature 2011, 479:74-79.

90. Colin J, Candelli T, Porrua O, Boulay J, Zhu C, Lacroute F et al.:

- Roadblock termination by reb1p restricts cryptic and readthrough transcription. Mol Cell 2014, 56:667-680.

Reb1p binding to DNA creates a roadblock that obstructs Pol II transcription and induces termination in yeast.

91. Aida M, Chen Y, Nakajima K, Yamaguchi Y, Wada T, Handa H: Transcriptional pausing caused by NELF plays a dual role in regulating immediate-early expression of the junB gene. Mol Cell Biol 2006, 26:6094-6104.

92. Kininis M, Isaacs GD, Core LJ, Hah N, Kraus WL: Postrecruitment regulation of RNA polymerase II directs rapid signaling responses at the promoters of estrogen target genes. Mol Cell Biol 2009, 29:1123-1133.

93. Danko CG, Hah N, Luo X, Martins AL, Core L, Lis JT et al: Signaling pathways differentially affect RNA polymerase II initiation, pausing, and elongation rate in cells. Mol Cell 2013, 50:212-222.

94. Gilchrist DA, Nechaev S, Lee C, Ghosh SKB, Collins JB, Li Let al: NELF-mediated stalling of Pol II can enhance gene expression by blocking promoter-proximal nucleosome assembly. Genes Dev 2008, 22:1921-1933.

95. Sun J, Li R: Human negative elongation factor activates transcription and regulates alternative transcription initiation. $J$ Biol Chem 2010, 285:6443-6452.

96. Gilchrist DA, Dos Santos G, Fargo DC, Xie B, Gao Y, Li L et al.: Pausing of RNA polymerase II disrupts DNA-specified nucleosome organization to enable precise gene regulation Cell 2010, 143:540-551.

97. Proshkin S, Rahmouni AR, Mironov A, Nudler E: Cooperation between translating ribosomes and RNA polymerase in transcription elongation. Science 2010, 328:504-508.

98. Saponaro M, Kantidakis T, Mitter R, Kelly GP, Heron M, Williams H

-• et al.: RECQL5 controls transcript elongation and suppresses genome instability associated with transcription stress. Cell 2014, 157:1037-1049.

Depletion of RECQL5 increases the global transcription elongation rate in human cells and also causes strong Pol II pausing as detected by ChIPseq. The study also reveals a link between transcriptional pausing during RECQL5 depletion and genome instability.

99. Pan T, Sosnick T: RNA folding during transcription. Annu Rev Biophys Biomol Struct 2006, 35:161-175.

100. Beyer AL, Osheim YN: Splice site selection, rate of splicing, and alternative splicing on nascent transcripts. Genes Dev 1988, 2:754-765

101. Carrillo Oesterreich F, Herzel L, Straube K, Hujer K, Howard J,

- Neugebauer KM: Splicing of nascent RNA coincides with intron exit from RNA polymerase II. Cell 2016, 165:372-381.

Combining long-read sequencing and targeted paired-end sequencing of nascent RNA reveals that a majority of splicing occurs co-transcriptionally and rapidly in yeast.

102. Eperon LP, Graham IR, Griffiths AD, Eperon IC: Effects of RNA secondary structure on alternative splicing of pre-mRNA: is 
folding limited to a region behind the transcribing RNA polymerase? Cell 1988, 54:393-401.

103. Roberts GC, Gooding C, Mak HY, Proudfoot NJ, Smith CW: Co-transcriptional commitment to alternative splice site selection. Nucleic Acids Res 1998, 26:5568-5572.

104. Cáceres JF, Kornblihtt AR: Alternative splicing: multiple control mechanisms and involvement in human disease. Trends Genet Elsevier 2002, 18:186-193 http://www.sciencedirect.com/ science/article/pii/S0168952501026269.

105. Naftelberg S, Schor IE, Ast G, Kornblihtt AR: Regulation of alternative splicing through coupling with transcription and chromatin structure. Annu Rev Biochem 2015, 84:165-198.

106. Saldi T, Cortazar MA, Sheridan RM, Bentley DL: Coupling of RNA polymerase II transcription elongation with pre-mRNA splicing. J Mol Biol 2016, 428:2623-2635.

107. de la Mata M, Alonso CR, Kadener S, Fededa JP, Blaustein M, Pelisch F et al:: A slow RNA polymerase II affects alternative splicing in vivo. Mol Cell 2003, 12:525-532.

108. Braberg H, Jin H, Moehle EA, Chan YA, Wang S, Shales M et al.: From structure to systems: high-resolution, quantitative genetic analysis of RNA polymerase II. Cell 2013, 154:775-788.

109. Dujardin G, Lafaille C, de la Mata M, Marasco LE, Muñoz MJ, Le Jossic-Corcos C et al.: How slow RNA polymerase II elongation favors alternative exon skipping. Mol Cell 2014, 54:683-690.

110. Fong N, Kim H, Zhou Y, Ji X, Qiu J, Saldi T et al.: Pre-mRNA splicing is facilitated by an optimal RNA polymerase II elongation rate. Genes Dev 2014, 28:2663-2676.

111. Alexander RD, Innocente SA, Barrass JD, Beggs JD: Splicingdependent RNA polymerase pausing in yeast. Mol Cell 2010, 40:582-593.

112. Proudfoot NJ: Transcriptional termination in mammals: stopping the RNA polymerase II juggernaut. Science 2016, 352 : aad9926.

113. Hazelbaker DZ, Marquardt S, Wlotzka W, Buratowski S: Kinetic competition between RNA polymerase II and Sen1-dependent transcription termination. Mol Cell 2013, 49:55-66.

114. Fong N, Brannan K, Erickson B, Kim H, Cortazar MA, Sheridan RM

-• et al.: Effects of transcription elongation rate and Xrn2 exonuclease activity on RNA polymerase II termination suggest widespread kinetic competition. Mol Cell 2015, 60:256-267.

Using Pol II elongation mutants, the authors show that slow Pol II mutants terminate earlier, whereas fast Pol II mutants terminate transcription later. This finding suggests that the zone of transcription termination depends on the rate of transcription elongation.

115. Lemay J-F, Larochelle M, Marguerat S, Atkinson S, Bähler J, Bachand F: The RNA exosome promotes transcription termination of backtracked RNA polymerase II. Nat Struct Mol Biol 2014, 21:919-926.

116. Park J, Kang M, Kim M: Unraveling the mechanistic features of

- RNA polymerase II termination by the $5^{\prime}-3^{\prime}$ exoribonuclease Rat1. Nucleic Acids Res 2015, 43:2625-2637.

In vitro transcription termination assays reveal that the misincorporation of nucleotides, which induces transcriptional pausing and backtracking, enhances termination by the Rat1/Rai1 complex.

117. Liu X, Kraus WL, Bai X: Ready, pause, go: regulation of RNA

- $\quad$ polymerase II pausing and release by cellular signaling pathways. Trends Biochem Sci 2015, 40:516-525.

This review article focuses on the relationship between cell signaling pathways and RNA polymerase II pausing, both under normal conditions and during disease development.

118. Rahl PB, Lin CY, Seila AC, Flynn RA, McCuine S, Burge CB et al.: C-Myc regulates transcriptional pause release. Cell 2010, 141:432-445.

119. Anand P, Brown JD, Lin CY, Qi J, Zhang R, Artero PC et al.: BET bromodomains mediate transcriptional pause release in heart failure. Cell 2013, 154:569-582.
120. Adelman K, Kennedy MA, Nechaev S, Gilchrist DA, Muse GW, Chinenov $Y$ et al.: Immediate mediators of the inflammatory response are poised for gene activation through RNA polymerase II stalling. Proc Natl Acad Sci U S A 2009, 106:18207-18212.

121. Levine M: Paused RNA polymerase II as a developmental checkpoint. Cell 2011, 145:502-511.

122. Larson DR, Zenklusen D, Wu B, Chao JA, Singer RH: Real-time observation of transcription initiation and elongation on an endogenous yeast gene. Science 2011, 332:475-478.

123. Raha D, Hong M, Snyder M: ChIP-Seq: a method for global identification of regulatory elements in the genome. Curr Protoc Mol Biol 2010:1-14. Chapter 21: Unit 21.19.

124. Jackson V: Studies on histone organization in the nucleosome using formaldehyde as a reversible cross-linking agent. Cell 1978, 15:945-954.

125. Solomon MJ, Larsen PL, Varshavsky A: Mapping protein-DNA interactions in vivo with formaldehyde: evidence that histone H4 is retained on a highly transcribed gene. Cell 1988, 53:937-947.

126. Rhee HS, Pugh BF: ChIP-exo method for identifying genomic location of DNA-binding proteins with near-single-nucleotide accuracy. Curr Protoc Mol Biol 2012. Chapter 21: Unit 21.24.

127. He Q, Johnston J, Zeitlinger J: ChIP-nexus enables improved - detection of in vivo transcription factor binding footprints. Nat Biotechnol 2015, 33:395-401.

This study introduces the ChIP-nexus approach. Application of this method to several transcription factors (TFs) in human and Drosophila cells reveals DNA binding sites at bp resolution and highlights the role of the DNA shape in TF binding.

128. Weber J, Jelinek W, Darnell JE Jr: The definition of a large viral transcription unit late in Ad2 infection of HeLa cells: mapping of nascent RNA molecules labeled in isolated nuclei. Cell 1977. 10:611-616.

129. Mahat DB, Kwak H, Booth GT, Jonkers IH, Danko CG, Patel RK

- et al.: Base-pair-resolution genome-wide mapping of active RNA polymerases using precision nuclear run-on (PRO-seq) Nat Protoc 2016, 11:1455-1476.

This paper provides a step-by-step protocol for the PRO-seq approach in several model systems, including Drosophila and mammalian cells.

130. Core LJ, Waterfall JJ, Gilchrist DA, Fargo DC, Kwak H, Adelman K et al.: Defining the status of RNA polymerase at promoters. Cell Rep 2012, 2:1025-1035.

131. Wang W, Carey M, Gralla JD: Polymerase Il promoter activation: closed complex formation and ATP-driven start site opening. Science 1992, 255:450-453.

132. Gilmour DS, Fan R: Detecting transcriptionally engaged RNA polymerase in eukaryotic cells with permanganate genomic footprinting. Methods 2009, 48:368-374.

133. Churchman LS, Weissman JS: Native elongating transcript sequencing (NET-seq). Curr Protoc Mol Biol 2012:1-17. Chapter 4: Unit 4.14.

134. Mayer A, Churchman LS: Genome-wide profiling of RNA

- polymerase transcription at nucleotide resolution in human cells with native elongating transcript sequencing. Nat Protoc 2016, 11:813-833.

This paper provides a detailed description, including a step-by-step guide, of the NET-seq approach for mammalian cells.

135. Nojima T, Gomes T, Carmo-Fonseca M, Proudfoot NJ:

- Mammalian NET-seq analysis defines nascent RNA profiles and associated RNA processing genome-wide. Nat Protoc 2016, 11:413-428.

This paper provides a step-by-step protocol for the mNET-seq approach using different Pol II antibodies. 